\title{
Heavy flavour production with the ATLAS detector at LHC
}

\author{
Vincenzo Canale on behalf of the ATLAS Collaboration ${ }^{\mathrm{a}, *}$ \\ ${ }^{a}$ Dipartimento di Fisica, Università di Napoli Federico II E Sezione INFN Napoli, \\ Complesso Universitario Monte S. Angelo, Via Cintia, 80126 - Napoli, Italy.
}

\begin{abstract}
We present some recent results on heavy flavour production obtained with ATLAS during the LHC runs in 2011-2012.
\end{abstract}

Keywords: heavy flavor, quarkonium, QCD production, ATLAS, LHC

\section{Introduction}

The production of heavy flavours (HF) at hadron colliders provides particular opportunity to study the theory of Quantum Chromodynamics (QCD), in particular the boundary of the perturbative and non-perturbative regimes. At $\mathrm{LHC}$, due to the high energy available $\sqrt{s}=(7 \div 8) \mathrm{TeV}$, it is possible to explore new kinematic regions (e.g., high ${ }^{1} p_{T}$ up to $\sim 10^{2} \mathrm{GeV}$ ) to test the predictions of various theoretical models for both quarkonium and open state production. In most cases, corrections over the leading order (LO) are available (e.g., next-to-leading (NLO), next-to-leading-log (NLL)) and an accurate quantitative comparison of data with QCD predictions can be performed to discriminate among various quarkonium production models (color singlet (CS) [1], color octet (CO or NRQCD) [2], color evaporation (CE) [3], $k_{T}$ factorization [4]).

\section{Experimental aspects}

\subsection{Detector performances}

ATLAS is a multipurpose apparatus to study $p p$ interactions at LHC, the detailed description and performances of the detector can be found in Ref. [5]. Regarding the analysis on HF during the LHC runs of 2011-2012 at $\sqrt{s}=(7 \div 8) \mathrm{TeV}$, the main detector components are: (a) the Muon System (MS) with tracking capability up to pseudorapidity $|\eta| \leq 2.7$, with momentum resolution

\footnotetext{
* Speaker

Email address: vincenzo.canale@na.infn.it (Vincenzo Canale on behalf of the ATLAS Collaboration)

${ }^{1} p_{T}$ is the transverse momentum to the direction of colliding beams.
}

Preprint submitted to Nuc. Phys. (Proc. Suppl.)
$\sigma_{p_{T}} / p_{T}=0.06$ up to $60 \mathrm{GeV}$; (b) the Inner Detector (ID) which reconstruct tracks up to $|\eta| \leq 2.5$ with efficiency $\epsilon_{t r k}=0.99$. The vertexing accuracy (primary and secondary) results in a resolution on the track impact parameter $^{2} \sigma_{\delta} \simeq 10 \mu \mathrm{m}$. The trigger for HF physics is based on di-muons up to $|\eta| \leq 2.4$ and with momentum thresholds $p_{T}^{(\mu)}(\mathrm{GeV})=4-6$.

The invariant mass distribution for a final state $Q$ is the key-tool for HF analyses; the typical resolutions are in the range $(50 \div 100) \mathrm{MeV}$ for $\mu \mu$ pairs or other exclusive states, and the observed signal/background ratios are high. From vertices reconstruction, it is possible to obtain the proper lifetime ${ }^{3}$ to reduce background or disentangle prompt and non-prompt ${ }^{4}$ components. For example, figure 1 shows the invariant mass distribution for $\psi(2 S) \rightarrow J / \psi(\mu \mu) \pi \pi$.

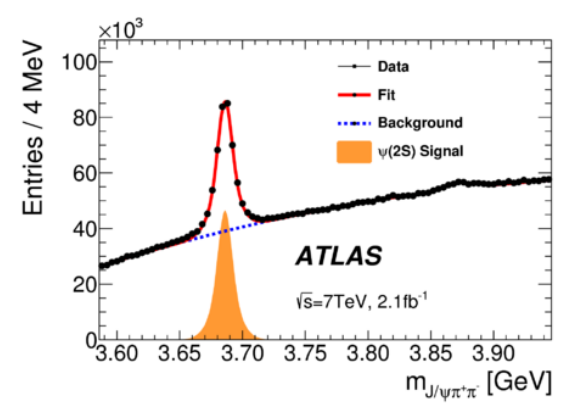

Figure 1: Mass distribution for $\psi(2 S) \rightarrow J / \psi(\mu \mu) \pi \pi$.

\footnotetext{
${ }^{2} \delta$ is the distance of closest approach to the primary vertex.

${ }^{3}$ The (pseudo) proper lifetime is $t=L_{T} \cdot \frac{m_{Q}}{P_{T}}$ where $L_{T}$ is transverse decay lenght projected along the $\vec{P}_{T}$ of the resonance.

${ }^{4}$ Non-prompt component originates from decays of of b-hadrons.
}

September 6, 2014 


\subsection{Cross section evaluation}

Experimentally, for each channel $Q \rightarrow$ final, the double differential cross section in $p_{T}$ and rapidity $y$, is obtained from

$$
\frac{d^{2} \sigma_{Q}}{d p_{t} d y} \cdot B R(Q \rightarrow \text { final })=\frac{N_{\text {corr }}^{Q \rightarrow \text { final }}}{\mathcal{L} \cdot \Delta p_{T} \cdot \Delta y},
$$

where $\mathcal{L}$ is the integrated luminosity ${ }^{5}, \Delta p_{T}(y)$ is the bin size of the differential variable, and $N_{c o r r}^{Q \rightarrow \text { final }}$ is the signal yield corrected for efficiency, acceptance and background subtraction. The extraction of the signals is obtained with unbinned maximum likelihood methods and the corresponding uncertainties are in general at the level of a few \%. The efficiency corrections for tracking, reconstruction and triggering are obtained with data driven methods (e.g., tag and probe for the trigger) in order to reduce the systematic uncertainties which amount typically to $(5 \div 10) \%$. The acceptance corrections, to recover the visible phase space in $p_{T}$ and $y$ for comparison with QCD predictions, are obtained with simulation. They depend on the unknown polarization status of $Q$ (spin alignment [6]). The correction is evaluated for the isotropic case and an eveloppe of variations is computed for the various polarizations (e.g., the $\psi(2 S) \rightarrow J / \psi \pi \pi$ state is shown in figure 2 as a function of $p_{T}$ ).

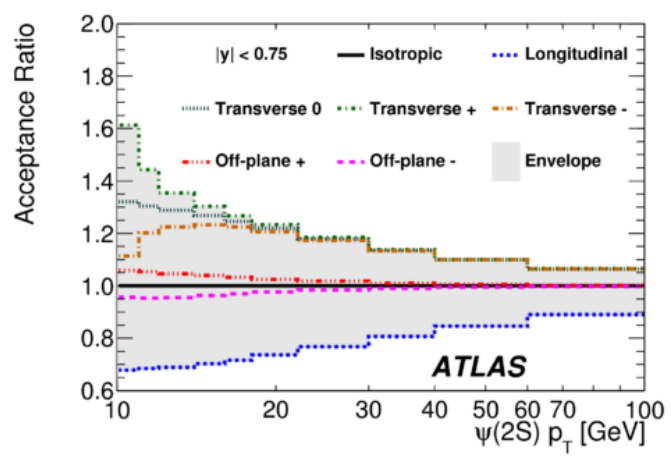

Figure 2: Acceptance correction ratios for $\psi(2 S) \rightarrow J / \psi \pi \pi$.

\section{Charmonium production}

First studies on prompt charmonium production were based on the measurement of $J / \psi$ [7]. Howwever, comparisons with theoretical models are difficult for $J / \psi$ due to the uncertain contributions from higher $(c \bar{c})$ states which decays to $J / \psi$ (feed-down effect).

\footnotetext{
${ }^{5}$ The $\mathcal{L}$ may vary for each analysis (trigger, etc...).
}

\section{1. $\psi_{2 S}$ and $\chi_{c n J}$ production}

To overtake feed-down uncertainties, analyses were performed to study the production of $\psi(2 S)$ [8] ( $\chi_{c n J}$ [9]) the S-wave (P-wave) just below the open charm threshold for which the feed down effect is absent (reduced). The decay channels are $\psi(2 S) \rightarrow$ $J / \psi\left(\mu^{+} \mu^{-}\right) \pi^{+} \pi^{-}$and $\chi_{c n J} \rightarrow J / \psi\left(\mu^{+} \mu^{-}\right) \gamma\left(e^{+} e^{-}\right)$; the prompt and non-prompt productions are disentangled using reconstruction of secondary vertices. The differential cross sections, as functions of $p_{T}$ for different $y$ intervals, for prompt production of $\psi(2 S)$ and $\chi_{c n J}$ are shown in figures 3 and 4 ; the predictions for different theoretical models are also reported.

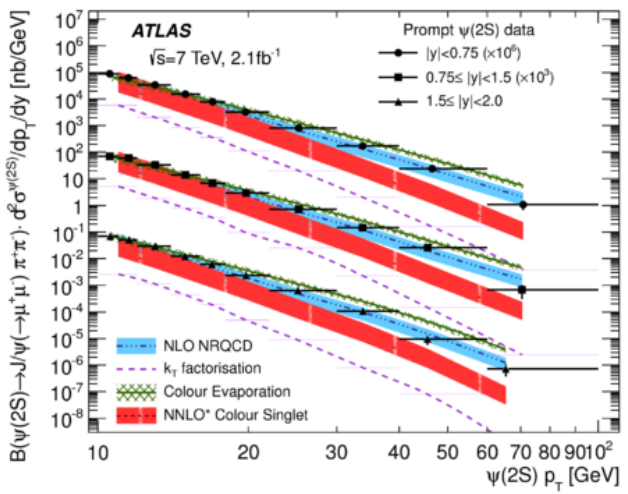

Figure 3: $p_{T}$-differential cross section for prompt $\psi(2 S)$ production.
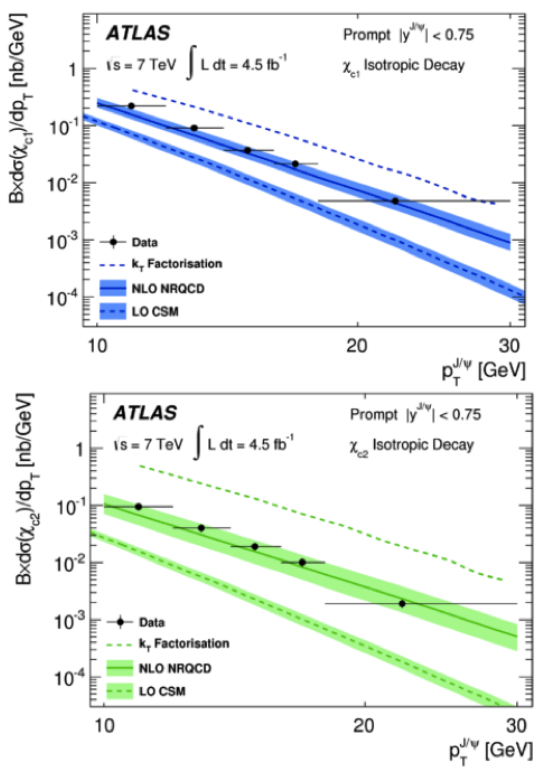

Figure 4: $p_{T}$-differential cross section for prompt $\chi_{c n J}$ production. 
A good agreement is observed with NRQCD (LO and NLO) at low and medium $p_{T}$, while in the new high $p_{T}$ regions some deviations appear. The CSM and $k_{T}$ models may need higher order contributions. For nonprompt production, $\psi(2 S)$ agrees with predictions at low $p_{T}$, but improvements are needed at higher $p_{T} ; \chi_{n J}$ is generally in good agreement with predictions but the range in $p_{T}$ is still limited.

\section{2. $W+J / \psi$ associated production cross section}

The study of associated production of prompt $J / \psi$ with vector bosons ( $W$ or $Z$ ) allows testing the specific QCD predictions [10] and, moreover, it is sensitive to physics in the Higgs sector [11]. ATLAS has performed the measurement [12] in the decay channels $J / \psi \rightarrow \mu^{+} \mu^{-}$ and $W^{ \pm} \rightarrow \mu^{ \pm} v_{\mu}$. For this analysis, the single muon trigger with $p_{T}^{(\mu)} \geq 18 \mathrm{GeV}$ was used, a standard selection for $J / \psi$ was applied disentangling prompt from nonprompt component with pseudo-proper lifetime. The $W$ candidates were selected applying tight muon selection ( $p_{T} \geq 25 \mathrm{GeV}$ and $|\eta| \leq 2.4$ ) associated with missing energy $E_{T}^{\text {miss. }} \geq 25 \mathrm{GeV}$. The separation of signal from multijet background is obtained with the $W$ transverse mass $m_{T}^{(W)}=\sqrt{2 p_{T}^{(\mu)} \cdot E_{T}^{\text {miss. }} \cdot\left(1-\cos \Delta \phi_{\mu \nu}\right)} \geq 40 \mathrm{GeV}$. The yield of prompt $J / \psi$ production associated with $W$, was estimated to $N_{J / \psi+W}=29.2_{-6.5}^{+7.2}$ of which $1.8 \pm 0.2$ originate from pile-up; the corresponding $p$-value of a background fluctuation is $2.1 \cdot 10^{-7}$ ( $5.1 \sigma$ significance). To compare with predictions the yield should be corrected for double parton scattering (DPS) contribution, which is estimated through

$$
d \sigma_{D P S}=d \sigma_{W} \cdot \mathcal{P}_{J / \psi \mid W}=\frac{d \sigma_{W} \cdot d \sigma_{J / \psi}}{\sigma_{e f f}},
$$

where $\sigma_{W}$ and $\sigma_{J / \psi}$ are from inclusive measurements and $\sigma_{e f f}$ is obtained from $W+2$ jets analysis [13]. The estimated contribution is $N_{D P S}=10.8 \pm 4.2$ events $(\sim$ $37 \%)$.

The experimental ratios $\frac{1}{\sigma_{W}} \frac{\Delta \sigma_{W+J / \psi}}{\Delta y}$, integrated over $J / \psi$ intervals of $p_{T}$ and $y$, are shown in figure 5. The result (fiducial) is corrected for $J / \psi$ acceptance (inclusive) and for DPS contribution (DPS-subtracted). Both NLO-CO and LO-CS model predictions are almost one order of magnitude below the experimental value but uncertainties are still large.

\section{Bottomonium production}

The study of bottomonium production complements the studies of charmonium systems. Due to the larger mass

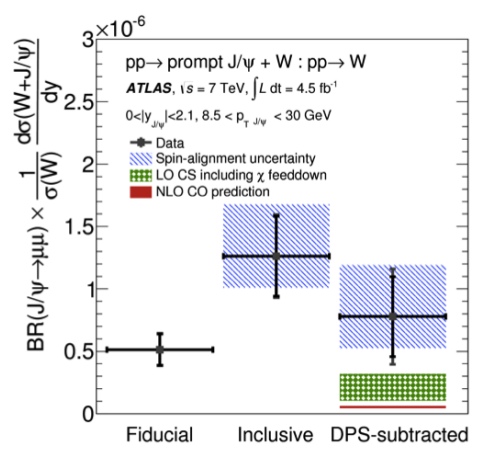

Figure 5: Ratio of cross section for $W+J / \psi$ production.

of the bottom quark compared to the charm quark, it allows more accurate theoretical calculations than in the charmonium family, which suffer from poorer perturbative convergence. The ATLAS analysis [14] on $\Upsilon_{n s}$ production, through the decay channel $\Upsilon \rightarrow \mu^{+} \mu^{-}$, extends the $p_{T}$ range of previous cross section result up to $70 \mathrm{GeV}$, where new production mechanisms may play a more important role [15], and the impact of the dependence of the production cross section on the spin alignment and feed-downs is reduced.

The selection cuts of $\mu \mu$ candidates are adaptated to the $\Upsilon$ mass region. The mass-resolution allows disentangling the yields for the states $\Upsilon(n S)$ for $n=1,2,3$. The yields are used to determine the cross section as a function of $p_{T}^{\Upsilon}$ and $y$.

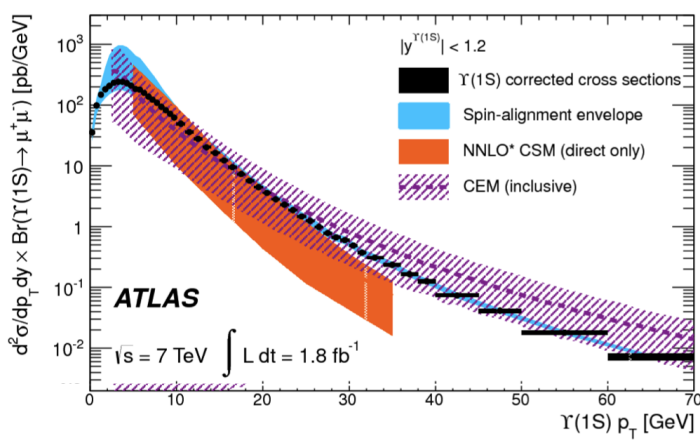

Figure 6: $p_{T}$-differential cross sesction for $\Upsilon(n S)$ production.

Figure 6 shows the $d \sigma_{\Upsilon(1 S)} / d p_{T}$ for $|y| \leq 1.2$ with the predictions of CEM and CSM. Similar behaviour is observed for all $\Upsilon$ 's, an important disagreement is observed in the new high- $p_{T}$ region where both feed-down and spin alignment effects are reduced. 


\section{Open B-state results}

\section{1. $B^{+}$production}

The NLO predictions for the b-hadron production crosssection have been improved with fixed order plus next-to-leading-logarithms (FONLL) calculations [16]. However, the dependence of the theoretical predictions on the factorisation and renormalisation scales and the b-quark mass results in theoretical uncertainties of up to $40 \%$. Therefore, it is important to measure the bhadron cross sections at high $\sqrt{s}$ and for B mesons of higher $p_{T}$. ATLAS measured [17] the $B^{+}$production $^{6}$ through the decay channel $B^{+} \rightarrow J / \psi\left(\mu^{+} \mu^{-}\right) K^{+}$up to $p_{T} \sim 100 \mathrm{GeV}$ and rapidity $|y|<2.25$.

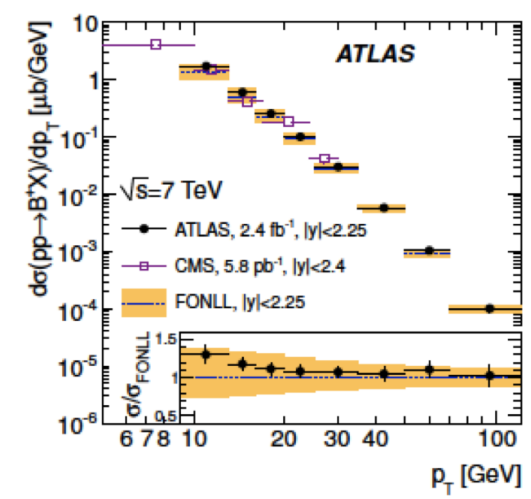

Figure 7: $p_{T}$-differential cross section for $B^{+}$production.

The measured $p_{T}$-differential cross-section for $B^{+}$production is reported in figure 7 with the some theoretical predictions (Powheg+Pythia and MC@NLO+Herwig generation models, and FONLL with $f_{\bar{b} \rightarrow B^{+}}=0.40 \pm$ 0.01). Within the theoretical uncertainties (scales and $m_{b}$ ), there is an agreement with the measured integrated cross-sections and with the dependence on $p_{T}$.

\subsection{Measurement of the $B R\left(B^{+} \rightarrow \chi_{c}+K^{+}\right)$}

ATLAS has measured [8] the $B R\left(B^{+} \rightarrow \chi_{c} K^{+}\right)$from the $\chi_{c} \rightarrow J / \psi \gamma$ sample and the reference $B^{+} \rightarrow J / \psi K^{+}$:

$$
B R\left(B^{+} \rightarrow \chi_{c} K^{+}\right)=\mathcal{A}_{B} \cdot \frac{N_{\chi_{c}}^{B}}{N_{J / \psi}^{B}} \cdot \frac{B R\left(B^{+} \rightarrow J / \psi K^{+}\right)}{B R\left(\chi_{c} \rightarrow J / \psi \gamma\right)}
$$

In (3), the BRs are taken from [18]; the acceptance ratio $\mathcal{A}_{B}$ for the channels, determined with simulation, benfits of the cancellation of correlated uncertainties.

\footnotetext{
${ }^{6}$ Results correspond to both charged states assuming $\sigma_{B^{+}}=\sigma_{B^{-}}$
}

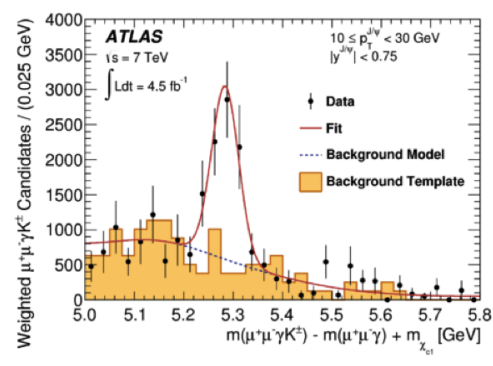

Figure 8: Mass distributions for $B^{+} \rightarrow \chi_{c} K^{+}$.

The signal yield is obtained from the mass distribution shown in figure 8 , and the value is

$$
B R\left(B^{+} \rightarrow \chi_{c} K^{+}\right)=(4.8 \pm 0.8[\text { stat. }] \pm 0.6[\text { syst. }]) \cdot 10^{-4}
$$

The result is in agreement with $(4.79 \pm 0.23) \cdot 10^{-4}[18]$ and competitive with the determinations at B-factories.

\section{Conclusions}

ATLAS performed several precision measurements on the HF production (charmonium, bottonium and $B^{+}$) at LHC to test various QCD models. These studies are very important because they refer to new kinematic regions (e.g., high $p_{T}$ up to $\sim 10^{2} \mathrm{GeV}$ ) and to channels less affected by uncertainties (e.g., $\psi(2 S)$ and $\chi_{c}$ ). ATLAS expects to exploit the full run-I and future run-II to probe new interesting phenomena in HF production (e.g., polarization, double quarkonium, associated production with $\mathrm{W}, \mathrm{Z}$ ).

\section{References}

[1] P. Artoisenet et al., Phys. Rev. Lett. 101 (2008) 152001

[2] Y.Q. Ma et al., Phys.Rev.Lett. 106 (2011) 042002

[3] J. F. Amundson et al., Phys. Lett. B 390 (1997) 323

[4] S. Baranov et al., Phys.Rev. D85 (2012) 014034

[5] ATLAS Collaboration, JINST 3 (2008) S08003

[6] P. Faccioli et al., Eur. Phys. Jour. C 69 (2010) 657

[7] ATLAS Collaboration, Nucl. Phys. B 850 (2011) 387444

[8] ATLAS Collaboration, arXiv:1407.5532, Accepted by JHEP

[9] ATLAS Collaboration, JHEP 07 (2014) 154

[10] G. Li et al., Phys. Rev. D 83 (2011) 014001 J.P. Lansberg and C. Lorce, Phys. Lett. B 726 (2013) 218

[11] G.T. Bodwin et al.,Phys. Rev. D 88 (2013) 053003 J.A. Grifols et al., Phys. Lett. B 197 (1987) 266

[12] ATLAS Collaboration, JHEP 04 (2014) 172

[13] ATLAS Collaboration, New Jour. of Phys. 15 (2013) 033038

[14] ATLAS Collaboration, Phys. Rev. D 87 (2013) 052004

[15] J. P. Lansberg, Eur. Phys. J. C 61 (2009) 693

[16] M. Cacciari et al., JHEP 1210 (2012) 137

[17] ATLAS Collaboration, JHEP 10 (2013) 042

[18] J. Beringer et al. (Particle Data Group), Phys. Rev. D86 (2012) 010001 . 